\title{
Capturing adaptive immunity against SARS-CoV-2 in patients with cancer
}

\author{
Cancer is associated with higher risk of severe COVID-19 outcomes. Two studies prospectively analyze the \\ immunological and clinical characteristics of a large cohort of patients with cancer following SARS-CoV-2 infection \\ or vaccination, providing important clinical insights to improve the management of such vulnerable patients.
}

\section{Hendrik Luxenburger and Robert Thimme}

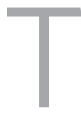
he COVID-19 pandemic has imposed a major global health burden, already accounting for more than 5.1 million deaths worldwide. Soon after the emergence of the pandemic, a global scientific effort facilitated the development of highly efficient vaccination strategies against the SARS-CoV-2 virus. Although recent studies have shown that $\mathrm{T}$ cells have an important role in sustained immunity following natural infection and vaccination ${ }^{1}$, the pathophysiology and determinants of SARS-CoV-2-specific immunity remain poorly characterized. Moreover, little is known about the strength and duration of the adaptive immune responses against SARS-CoV-2 in vulnerable individuals such as patients with cancer. Another key open question is the effect of emerging variants of concern (VOCs) on the virus-specific adaptive immunity triggered by infection or vaccination in these patients. The efficacy of the currently available vaccines in vulnerable patient populations is of critical medical importance and carries imminent implications for future vaccination strategies, including the evaluation of potential vaccination boosters. In this issue of Nature Cancer, Turajlic and colleagues characterize the specific adaptive immunity induced by full vaccination ${ }^{2}$ or infection ${ }^{3}$ with SARS-CoV-2, including by VOCs, in two reports from the CAPTURE (COVID-19 antiviral response in a pan-tumor immune monitoring) prospective study.

In these two elegant studies, the authors characterized the specific adaptive immunity against SARS-CoV-2 in patients with solid and hematological malignancies. Their first important finding is that natural infection and vaccination induce different immune profiles in people with solid versus hematological cancers ${ }^{2,3}$. Indeed, in line with previous studies ${ }^{4}$, they found that most patients with solid cancers have detectable S1-specific immunoglobulin $\mathrm{G}$ molecules, neutralizing antibodies and virus-specific $\mathrm{T}$ cell responses following natural infection and/or vaccination. In comparison, individuals with hematological malignancies show weaker cellular and humoral immune responses. Importantly, patients with either solid or hematological cancers show impaired immune responses compared to the general population, as indicated by lower seroconversion rates following natural infection or vaccination ${ }^{2,3}$ (Fig. 1a). At the cellular level, the CAPTURE study found a lower proportion of patients with detectable virus-specific $\mathrm{CD} 4^{+} \mathrm{T}$ cells ( $81 \%$ of patients tested among those with solid cancer and $58 \%$ in hematological cancer) and CD8 ${ }^{+} \mathrm{T}$ cells (51\% in solid cancer and $42 \%$ in hematological cancer) after natural infection ${ }^{3}$. This contrasts with healthy participants, who display rapid induction and then prolonged contraction of SARS-CoV-2-specific $\mathrm{CD}^{+} \mathrm{T}$ cells followed by the emergence of fully functional memory $\mathrm{CD} 8^{+} \mathrm{T}$ cells $\mathrm{s}^{5}$. A similar differential immune profile was also evident after vaccination, in which patients with cancer showed lower levels of virus-specific T cells (59\% after prime versus $79 \%$ after boost) compared to the general population, as seen in a previous study demonstrating the induction of fully functional $\mathrm{CD} 8^{+} \mathrm{T}$ cells in all tested immunocompetent patients after boost vaccination $^{2,6}$. However, the mechanisms responsible for the impairment of adaptive immunity in patients with both solid and hematological cancers are currently not well understood and most likely manifold. Overall, this impaired adaptive immunity in patients with cancer confirms that this vulnerable group might benefit from a third vaccination dose for adequate protection against SARS-CoV-2 infection. Consistent with this hypothesis, Shroff et al. ${ }^{7}$ recently reported that in patients with solid tumors, neutralizing antibodies are increased after a third vaccination booster, whereas levels of circulating spike-specific $\mathrm{T}$ cells are not. This interesting observation should be validated in patients with hematologic malignancies, given that immune responses in this group are more impaired.

The authors' second important observation in these two studies is the impact of specific cancer subtypes on the status of the adaptive immune system after natural infection or vaccination ${ }^{2,3}$. For example, leukemia is associated with impaired $\mathrm{T}$ cell responses, whereas patients with lymphoma display low antibody titers (Fig. 1d). Interestingly, each of these impaired immune responses was compensated by the other arm of the adaptive immune system, resulting, for instance, in higher virus-specific T cell responses in the absence of antibodies.

The authors also found a correlation between specific cancer treatments and SARS-CoV-2-specific immune responses. For example, patients given checkpoint inhibitor therapy show an impaired immune response after natural infection ${ }^{3}$. In this cohort, the levels of SARS-CoV-2-specific $\mathrm{CD}^{+} \mathrm{T}$ cells were reduced, whereas $\mathrm{CD} 8^{+}$ $\mathrm{T}$ cells levels remained comparable to those in the healthy control population. After vaccination, however, unlike in natural infection, checkpoint inhibitor therapy did not lead to impaired SARS-CoV-2-specific $\mathrm{CD}^{+} \mathrm{T}$ cell levels (Fig. 1d) ${ }^{2}$.

Another example illustrated in the CAPTURE study is the effect of anti-CD20 therapy on SARS-CoV2-specific immunity: such therapy was associated with impaired humoral immune responses to both natural infection and vaccination $^{2,3}$. Similar findings have been reported for patients with multiple sclerosis ${ }^{8}$, and in this case the impaired humoral immunity was compensated by cellular immune responses (Fig. 1d). The long-term presence of virus-specific $\mathrm{T}$ cell responses in patients without detectable antibodies has recently been described in patients with solid tumors ${ }^{7}$, as well as 
a

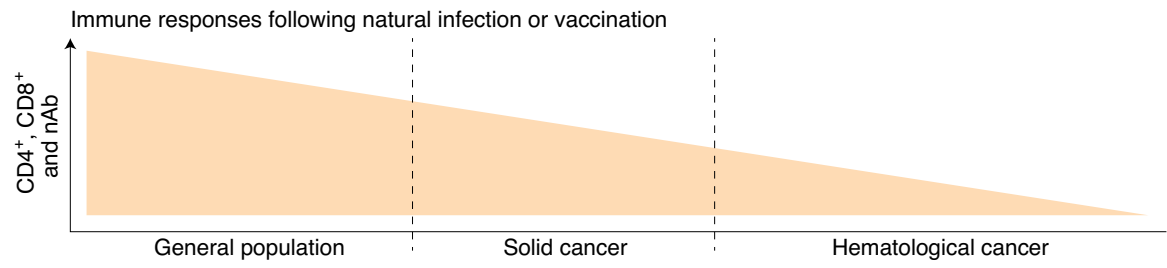

b

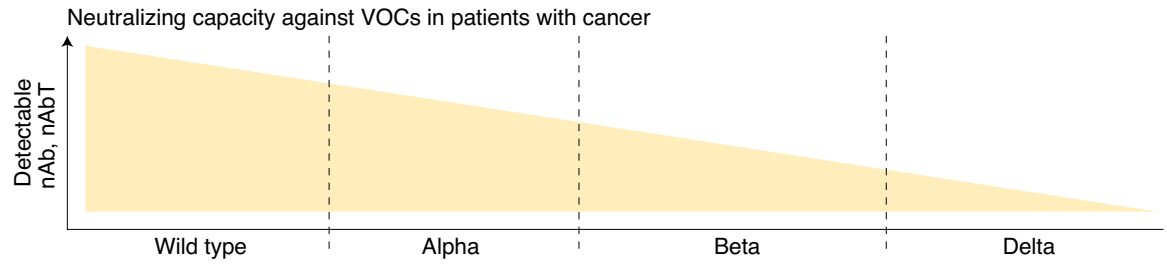

C

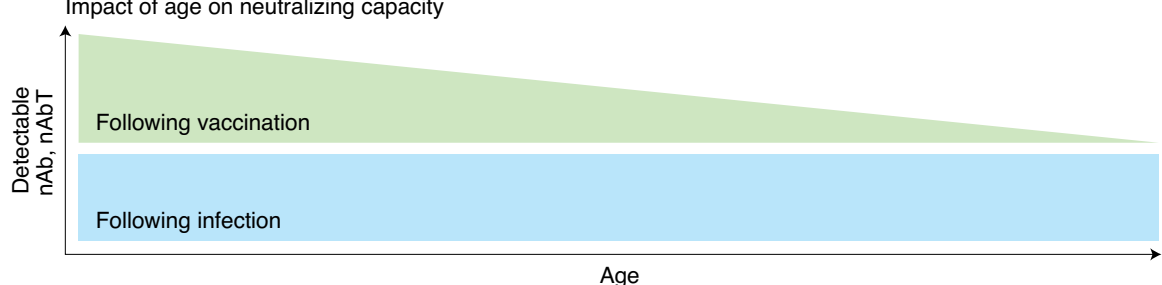

d

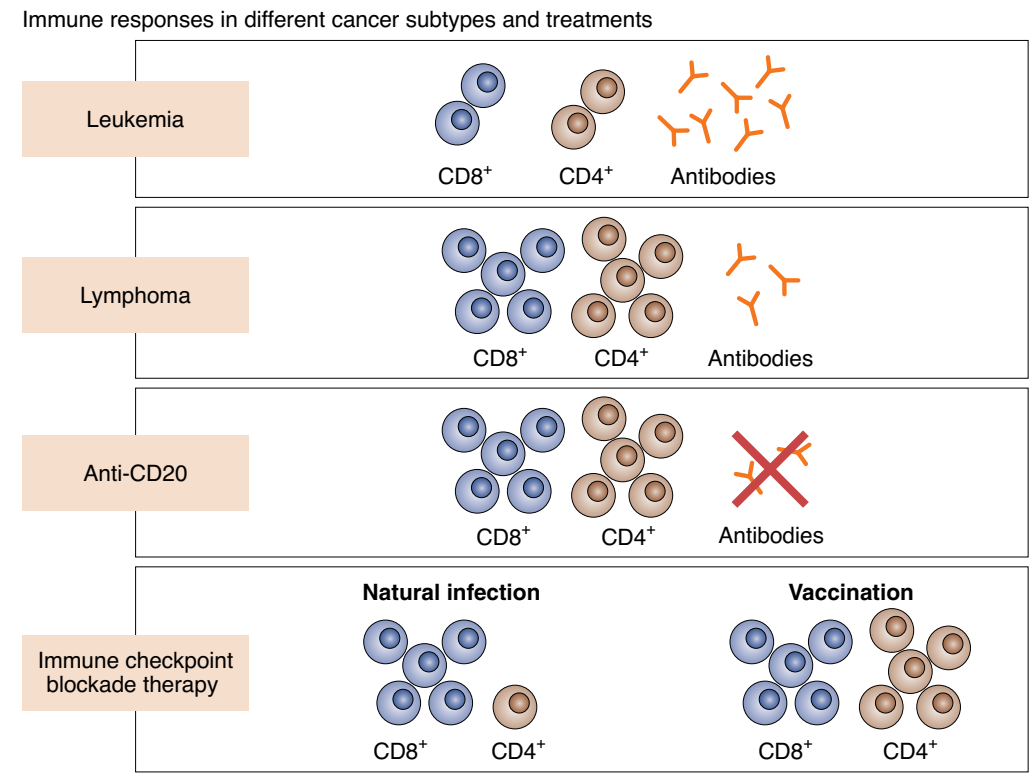

Fig. 1 | Humoral and cellular immunity in patients with solid and hematological cancers. a-d,

Impaired immune responses in cancer patients compared to the general population with induction of different immune profiles in solid versus hematological cancers after natural infection and vaccination (a). The neutralizing capacity against VOCs is reduced in patients with cancer (b), and neutralizing antibody titers against VOCs after vaccination are reduced in older individuals (c). Cancer subtypes and treatment modalities have implications for the SARS-CoV-2-specific immune response $(\mathbf{d}) . \mathrm{CD}^{+}, \mathrm{CD}^{+} \mathrm{T}$ cells; $\mathrm{CD} 8^{+}, \mathrm{CD} 8^{+} \mathrm{T}$ cells; $\mathrm{nAb}$, neutralizing antibodies; $\mathrm{nAbT}$, neutralizing antibody titers.

those with hematological malignancies ${ }^{9}$. The CAPTURE study extends this observation to patients with hematological malignancies and thus underlines the important role of SARS-CoV-2-specific $\mathrm{T}$-cell-mediated immunity in the absence of functional antibodies. protection in the context of circulating VOCs, which is a key finding given that VOC Delta is currently the predominant variant worldwide. Turajlic and colleagues demonstrated in patients with solid and hematological tumors that following infection with wild-type SARS-CoV-2, neutralizing antibodies against the wild-type virus were detectable in higher proportions of patients than those against the VOCs Alpha, Beta and Delta ${ }^{3}$. After prime vaccination, the median titers of neutralizing antibodies were below the detection limit for all viral strains in infection-naive patients with cancer. In this cohort, titers of neutralizing antibodies against wild-type SARS-CoV-2 were detectable at lower levels than those against the VOCs after boost vaccination. Of note, in patients with cancer who had had prior SARS-CoV-2 infection, neutralizing antibody titers against the wild type and VOCs were significantly higher after vaccination than those in infection-naive patients ${ }^{2}$.

Overall, the observation of reduced neutralizing antibody activity against VOCs in patients with cancer is in line with several studies in healthy individuals showing significantly weaker neutralizing activity against VOCs (as compared to wild-type virus) following either natural infection with wild-type SARS-CoV-2 or vaccination ${ }^{6,10-13}$. The reduced neutralizing capacity against VOCs in both patients with cancer and healthy individuals has direct clinical implications in regard to the potential implementation and prioritization of therapies based on monoclonal antibodies or convalescent plasma from patients infected with wild-type SARS-CoV-2, given that these might be less efficacious in patients infected with VOCs ${ }^{13,14}$.

Last, the authors observed that the age of cancer patients has implications for the SARS-CoV-2-specific humoral immune response. Indeed, whereas in natural infection, age was not associated with lower levels of neutralizing antibodies or the absence of virus-specific T cells, older age was significantly associated with reduced neutralizing antibody titers against all circulating VOCs after vaccination (Fig. $1 c)^{2,3}$. This observation is in line with previous studies in healthy patients ${ }^{15}$ and supports the idea that both older patients with cancer and older healthy individuals might potentially benefit from an early third vaccination booster.

Collectively, the CAPTURE study provides important insights into the humoral and cellular immunity found in the vulnerable group of patients with cancer. To validate the interesting and relevant findings by Turajlic and colleagues ${ }^{2,3}$, further studies 
in a larger, separate cohort will be required. Moreover, it will be of great interest to determine the phenotypical, functional and transcriptional characteristics of virus-specific $\mathrm{T}$ cells in patients with cancer in order to better understand the long-term immunity against SARS-CoV-2 infection following natural infection versus vaccination in this special patient cohort. Overall, the authors' important observations suggest that patients with cancer, especially older patients and those with hematological cancers, might benefit from an early boost vaccination, reflecting the urgent need to prioritize additional booster vaccination campaigns in vulnerable populations.
Hendrik Luxenburger (D) ${ }^{1,2}$ and

\section{Robert Thimme}

${ }^{1}$ Department of Medicine II (Gastroenterology, Hepatology, Endocrinology and Infectious Diseases), Freiburg University Medical Center, Faculty of Medicine, University of Freiburg, Freiburg, Germany. ${ }^{2} I M M-P A C T$, Faculty of Medicine, University of Freiburg, Freiburg, Germany.

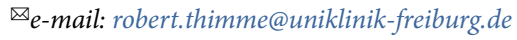

Published online: 16 December 2021

https://doi.org/10.1038/s43018-021-00298-2

References

1. Sette, A. \& Crotty, S. Cell 184, 861-880 (2021).

2. Fendler, A. et al. Nat. Cancer https://doi.org/10.1038/s43018-02100274-w (2021).
3. Fendler, A. et al. Nat. Cancer https://doi.org/10.1038/s43018-02100275-9 (2021).

4. Thakkar, A. et al. Cancer Cell 39, 1081-1090.e2 (2021).

5. Schulien, I. et al. Nat. Med. 27, 78-85 (2021).

6. Oberhardt, V. et al. Nature 597, 268-273 (2021).

7. Shroff, R. T. et al. Nat. Med. https://doi.org/10.1038/s41591-02101542-z (2021).

8. Zabalza, A. et al. Eur. J. Neurol. 28, 3384-3395 (2021).

9. Bange, E. M. et al. Nat. Med. 27, 1280-1289 (2021).

10. Wang, Z. et al. Nature 592, 616-622 (2021).

11. Planas, D. et al. Nature 596, 276-280 (2021).

12. Wall, E. C. et al. Lancet 398, 207-209 (2021)

13. Hoffmann, M. et al. Cell 184, 2384-2393.e12 (2021).

14. Wang, P. et al. Nature 593, 130-135 (2021).

15. Li, J. et al. Nat. Med. 27, 1062-1070 (2021).

Competing interests

The authors declare no competing interests. 